\title{
RD on Multi-TeV Linear Collider, Status and Perspectives
}

\section{Roberto Corsini*}

CERN, Switzerland

E-mail: roberto.corsini@cern.ch

\begin{abstract}
A high luminosity $\left(10^{34}-10^{35} \mathrm{~cm}^{2} / \mathrm{s}\right)$ electron-positron Collider (CLIC) with a nominal centreof-mass energy of $3 \mathrm{TeV}$ has been under study for a number of years in the framework of an international collaboration of laboratories and institutes, with the aim to provide the HEP community with a new facility for the post-LHC era. After a brief description of a CLIC scheme to extend Linear Colliders into the Multi- $\mathrm{TeV}$ colliding beam energy range, the main challenges and the very promising results already achieved will be presented. The presentation will mainly focus on the new Test Facility (CTF3) presently under construction at CERN in order to address the main key issues and demonstrate the feasibility of the CLIC technology before 2010.
\end{abstract}

International Europhysics Conference on High Energy Physics

July 21st - 27th 2005

Lisboa, Portugal

\footnotetext{
* Speaker.
} 


\section{The CLIC challenges, experimental results and planned tests}

The CLIC (Compact Linear Collider) design [1] is optimised for $3 \mathrm{TeV}$ but can be built in stages, increasing its energy without major modifications. To limit its total length, CLIC uses normal conducting accelerating structures operating at a very high gradient $(150 \mathrm{MV} / \mathrm{m})$, and powered by high power (150 MW) $30 \mathrm{GHz}$ RF pulses. Conventional RF sources do not cover this range and CLIC relies upon a two-beam-acceleration concept [2]. A high current electron beam (drive beam) runs parallel to the main beams and is decelerated to produce the RF power. The generation of high-intensity drive beam pulses with the right time structure is one of the main challenges in CLIC. A long pulse is accelerated in low frequency (937 MHz) normal-conducting accelerating cavities, operated in the "full beam loading" condition where the beam extracts more than $95 \%$ of the RF power. The electron bunches are then interleaved in stages by transverse RF deflectors in delay lines and rings to give the beam the desired bunch structure while increasing its intensity. The bunch spacing is reduced from $64 \mathrm{~cm}$ to $2 \mathrm{~cm}$, and the current increased from $5.7 \mathrm{~A}$ to $180 \mathrm{~A}$.

It is generally accepted that CLIC is the only viable technology for multi-TeV colliders. However, critical issues still need to be addressed. In the past years, the International Linear Collider Technical Review Committee [3] has listed a number of crucial items to prove CLIC feasibility (the so-called R1 items), and to arrive at a conceptual design (the R2 items). The experimental program of the new CLIC Test Facility (CTF3) deals with all the CLIC technology-related R1 and R2 issues, while issues common to all linear collider are investigated in the framework of the EuroTeV collaboration. The goal is to get an answer on the CLIC feasibility before 2010. By then the first LHC results should be available and the energy required for a future linear collider better known.

CTF3 [4] is presently being built and commissioned in stages at CERN, by an international collaboration including Ankara University, BINP, CIEMAT, Dapnia-Saclay, INFN-Frascati, LALOrsay, LAPP-Annecy, North Western University of Illinois, RAL, SLAC, Uppsala University and Finnish industry, while other institutes have shown interest in joining CTF3. CTF3 uses the buildings of the former LEP pre-injector complex (LPI) and makes maximum use of existing hardware. The experimental program will cover the following points: 1) Test of a prototype CLIC accelerating structure (including design features to damp higher order modes) at design gradient and pulse length (R1). 2) Validation of the drive beam generation scheme with a fully-loaded linac (R1). 3) Design and test of an adequately damped power-extraction structure, which can be switched on and off (R1). 4) Validation of beam stability and losses in the drive beam decelerator, and design of a machine protection system (R2). 5) Test of a relevant linac sub-unit with beam (R2).

Many experimental results have been obtained in the past years, which have guided and confirmed the technical choices undertaken. The feasibility of two-beam acceleration was already demonstrated in the CLIC Test Facility CTF2. There, an electron bunch was accelerated by 60 $\mathrm{MeV}$ using a string of $30 \mathrm{GHz}$ structures powered by a drive beam. Such drive beam, produced directly from a photocathode gun, had however a total charge and pulse length much lower than the nominal CLIC values. CTF2 has also been essential as a test bed for CLIC components. Its last two years of operation were indeed dedicated to testing of accelerating structures after damage to their copper irises was observed at high field. New structure designs, aimed at reducing the peak surface field for a given accelerating gradient, were explored. A big improvement in performance was obtained by employing irises made of arc-resistant materials like tungsten or molybdenum (see 
Figure 1). Eventually, an accelerating gradient of $190 \mathrm{MV} / \mathrm{m}$ was reached using molybdenum irises [5]. The RF pulse length was however limited by the drive beam injector to $16 \mathrm{~ns}$, well below the CLIC nominal value (70 ns). CTF3 is meant to extend these investigations to longer pulse lengths, up to and beyond the nominal value.

The technical feasibility of increasing the drive beam current by bunch interleaving by RF deflectors was successfully demonstrated in 2001 and 2002 at low current $(0.3 \mathrm{~A})$, using the modified LPI complex, during the so-called preliminary phase of CTF3 [6] (see Figure 1). Up to five bunch trains were combined over five turns in a combiner ring to reach $1.5 \mathrm{~A}$, without measurable losses. One of the aims of the new CTF3 programme is to test this process with long, high current pulses.
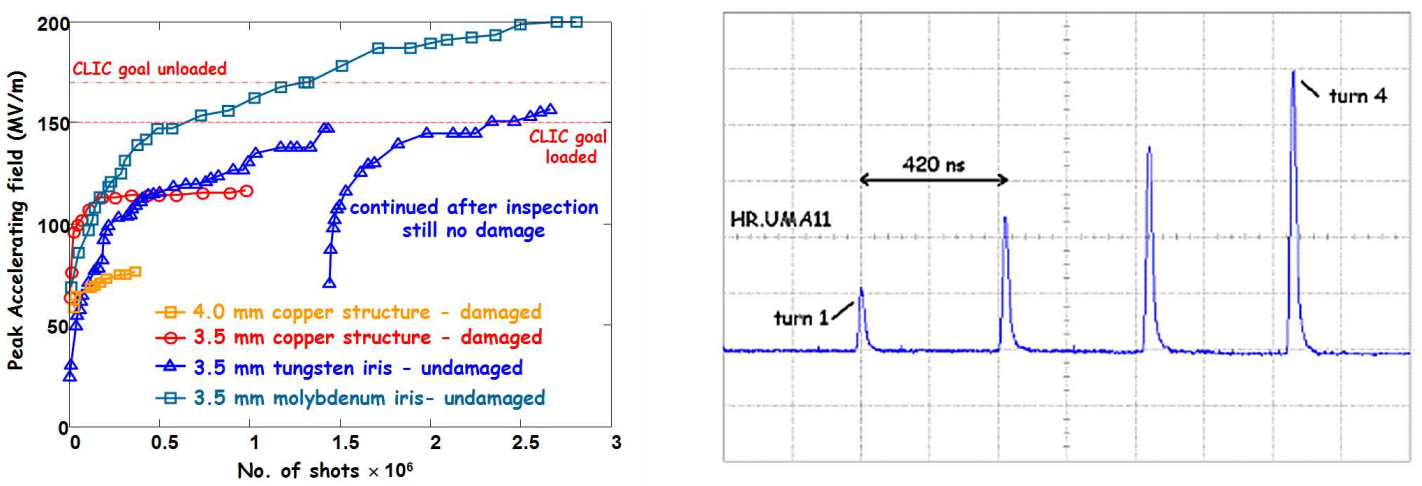

Figure 1: Previous results: RF conditioning history of several accelerating structures, with irises made of different materials and with different apertures, tested with beam in CTF2 (left). Oscilloscope trace from an intensity monitor in the CTF3 preliminary phase combiner ring, showing the circulating beam current for a beam combination test of factor four. The beam corrent increases over four turns from $0.3 \mathrm{~A}$ to $1.2 \mathrm{~A}$. (right)

\section{Status of the CTF3 complex}

The CTF3 complex is designed to work at a lower beam current and a lower energy than foreseen for the CLIC drive beam (3.5 A instead of 5.7 A and $150 \mathrm{MeV}$ instead of $2.4 \mathrm{GeV}$ ) [4]. In its final configuration (see Figure 2) CTF3 will be composed of a $70 \mathrm{~m}$ long drive-beam linac followed by a $42 \mathrm{~m}$ delay loop and and a $84 \mathrm{~m}$ ring, where the bunch manipulations will be carried out. After combination the drive beam will have a current of $35 \mathrm{~A}$, and will be transported to an experimental area to produce $30 \mathrm{GHz}$ RF power in a high power test stand. In the same area a separate linac will provide a main beam for a representative CLIC two-beam module, and a test decelerator will be dedicated to drive beam stability studies. The installation also includes a second high power test stand (halfway along the linac) working at a lower beam current, needed to continue RF component testing during installation and commissioning.

The project is proceeding as planned, alternating installation and commissioning periods. In 2003 and 2004, the injector, the linac with its $30 \mathrm{GHz}$ power production line and an end-of-linac chicane and instrumentation section have been installed and commissioned. In 2005, the installation of the delay loop was completed. The combiner ring and the experimental area will be put in operation in 2006 and 2007. In the linac, the design beam current and pulse length were reached rapidly. The beam was remarkably stable and no sign of beam break-up was observed at high cur- 


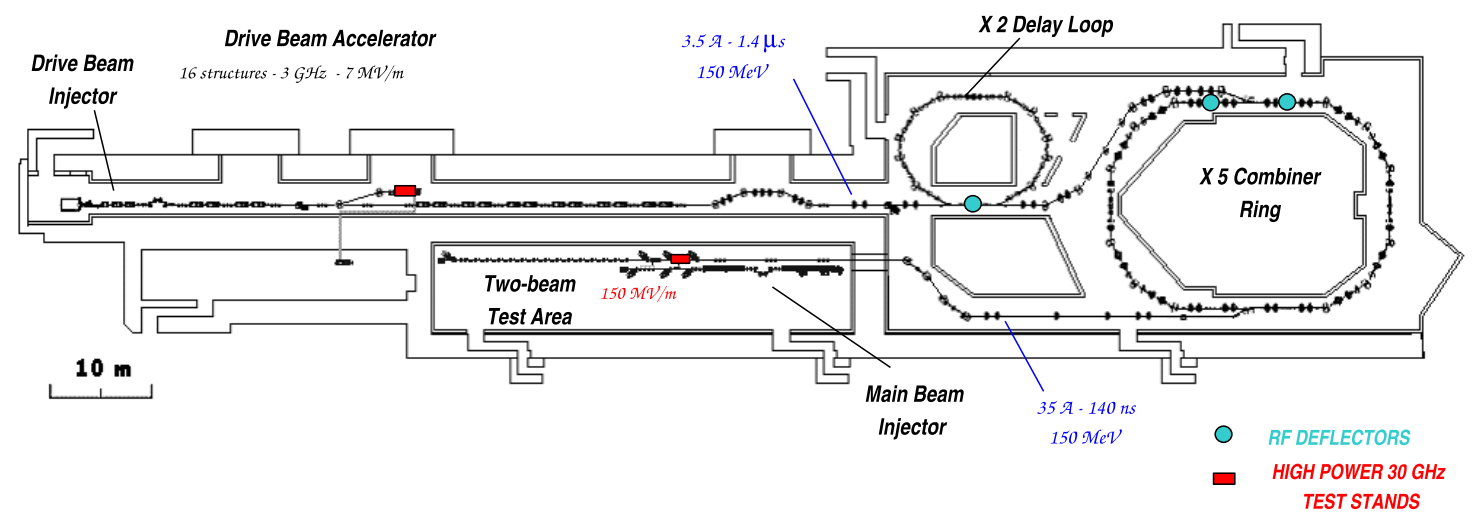

Figure 2: CTF3 layout.

rent, successfully demonstrating the operation under under full beam loading of the linac structures with their novel damping scheme [7]. The RF-to-beam efficiency was evaluated from input and output RF signals and confirmed by beam energy gain measurements. For a 4 A beam, an efficiency of $94 \%$ was obtained, in agreement with simulations. Several beam measurements were performed in the end-of-linac chicane, built by INFN/LNF, varying its optics to obtain different momentum compaction values, both positive and negative. A vertically deflecting RF structure was used together with an OTR screen to make bunch length measurements. Initial measurements showed bunch compression to less than $0.5 \mathrm{~mm}$ rms for an initial bunch length of $2 \mathrm{~mm}$. Comparison with the MAD model showed good agreement. Commissioning of the delay loop, also built by INFN/LNF, started recently, with the first beam circulating in November 2005.

CTF3 has started its role as a $30 \mathrm{GHz}$ power production facility with the first accelerating structure tests in 2005. These are ongoing, and at present RF pulses up to $90 \mathrm{MW}$ have been generated. Preliminary measurements shows that about $60 \mathrm{MW}$ were delivered to a molybdenum iris test structure, corresponding to a gradient in the first cell above $150 \mathrm{MV} / \mathrm{m}$. The structure has been conditioned up to now to a pulse length of $60 \mathrm{~ns}$, close to the CLIC nominal value.

\section{References}

[1] CLIC Study Team, Ed. G. Guignard, A 3 TeV e+e-linear collider based on CLIC technology, CERN 2000-008 and I. Wilson, The compact linear collider CLIC, Physics Reports 404-404 (2004) 365-378.

[2] H. Braun et al., Ed. R. Corsini, The CLIC RF power source, CERN 99-06, 1999.

[3] Editor G. Loew, International linear collider technical review committee second report, SLAC-R-606.

[4] G. Geschonke and A. Ghigo Eds, CTF3 Design Report, CERN/PS 2002-008 (RF).

[5] W. Wuensch, C. Achard, S. Döbert, H. H. Braun, I. Syratchev, M. Taborelli, I. Wilson, A Demonstration of High Gradient Acceleration, CERN-AB-2003-048-RF, Proc. PAC 2003.

[6] R. Corsini, A. Ferrari, L. Rinolfi, P. Royer, F. Tecker, Experimental results on electron beam combination and bunch frequency multiplication, Phys. Rev. ST Accel. Beams 7, 040101 (2004).

[7] E. Jensen, CTF3 Drive Beam Accelerating Structures, Proc. LINAC 2002, 9-23 August 2002, Gyeongju (Kyongju), Korea, CERN/PS 2002-068 (RF) and CLIC Note 538. 